\title{
O IMPACTO AMBIENTAL DA INTRODUÇÃO DE AUTOMÓVEIS DIESEL
}

\author{
FORCETTO, A. L. S. ${ }^{1}$, ABRANTES, R. $\mathrm{de}^{2}$ \\ ${ }^{1,2}$ CETESB - Companhia Ambiental do Estado de São Paulo \\ E-mails: $\underline{\text { aforcetto@sp.gov.br, rabrantes@sp.gov.br }}$
}

\section{RESUMO}

Enquanto na Europa uma grande quantidade de veículos leves de passageiros (VLP) utiliza o óleo Diesel como combustível, no Brasil o uso em VLP é proibido. No entanto, está em tramitação no Congresso Nacional Projeto de Lei 1013/2011, que extingue esta proibição, baseado nas premissas que o automóvel Diesel terá menor emissão de $\mathrm{CO}_{2}$ e menor poluição. Assim, o objetivo deste trabalho é fazer uma avaliação do impacto ambiental da emissão de gases poluentes e $\mathrm{CO}_{2}$ que haveria em metrópoles como a Região Metropolitana de São Paulo (RMSP), caso seja liberado o uso de óleo Diesel em VLP. Este estudo baseia-se nos resultados dos ensaios de homologação de veículos que possuem motorização similar Otto (gasolina e flex) e Diesel, bem como em dados de estudos de campo, analisados segundo a metodologia da CETESB para o cálculo do inventário de emissões veiculares. Os resultados apontam, para o automóvel Diesel em relação aos Otto, para forte aumento nas emissões de NOx e pequena redução na emissão de $\mathrm{CO}_{2}$ total, porém com aumento significativo do $\mathrm{CO}_{2}$ fóssil (descontados os biocombustíveis), resultados que são multiplicados ao se considerar as emissões reais de NOx e ao se projetar o impacto ambiental sobre a RMSP.

\section{INTRODUÇÃO}

No Brasil o óleo Diesel recebe menor carga tributária em relação à gasolina, com o objetivo de reduzir os custos do frete de mercadorias por caminhões e das tarifas de ônibus para o transporte urbano de massa [1]. Deste modo, os veículos leves de passageiros (VLP) são proibidos por lei de o utilizarem como combustível, situação oposta a da Europa, onde há significativa presença de VLP movidos a Diesel nas ruas.

Está em tramitação no Congresso Nacional o Projeto de Lei (PL) 1013/2011 [2], que libera a venda de automóveis Diesel no Brasil, surgindo daí a polêmica: os defensores do PL argumentam que o veículo Diesel é mais eficiente, portanto tem menor consumo e menor emissão de $\mathrm{CO}_{2}$. Quanto à emissão de poluentes, a alegação é que os veículos com sistemas antipoluição são pouco poluentes e assim trariam pouco impacto ambiental para o nosso país. Esta posição é contraposta pela Companhia Ambiental do Estado de São Paulo (CETESB), Organização Mundial da Saúde (OMS) e o Conselho Internacional para Transporte Limpo (International Council on Clean Transportation - ICCT) [3, 4, 5], entre outras instituições, assim vê-se que o assunto é polêmico, mas deve ser discutido à luz dos fatos, para que se tenha uma visão mais precisa. 
Deste modo, o objetivo deste artigo é avaliar qual o impacto ambiental haveria na Região Metropolitana de São Paulo (RMSP) quanto à geração de poluentes e de gases de efeito estufa, caso seja liberado a venda de automóveis Diesel no Brasil.

\section{OS VEÍCULOS COMO FONTE DE POLUIÇÃO ATMOSFÉRICA}

A RMSP, semelhante a outros grandes centros urbanos, está sujeita aos problemas relacionados à poluição, como a degradação do solo, água e ar. Desde o final dos anos 1960 começaram a surgir na imprensa relatos de episódios agudos de poluição do ar, que levou o governo paulista a criar em 1972 a rede de monitoramento da qualidade atmosférica, que fica sob a responsabilidade da Companhia Ambiental do Estado de São Paulo (CETESB) e faz o controle de material particulado inalável $\left(\mathrm{MP}_{10}\right.$ e $\left.\mathrm{MP} \mathrm{P}_{2,5}\right)$, ozônio $\left(\mathrm{O}_{3}\right)$, óxidos de nitrogênio ( $\mathrm{NOx}$ - combinação de $\mathrm{NO}$ e $\mathrm{NO}_{2}$ ), monóxido de carbono $(\mathrm{CO})$ e hidrocarbonetos (HC), além de diversos parâmetros meteorológicos [6].

As principais fontes de poluição atmosférica são os processos industriais e a frota veicular, com participação também da queima de biomassa, processos de ressuspensão de particulado e emissões com origem na cadeia de produção e distribuição de combustível [7], como mostra a Figura 1.

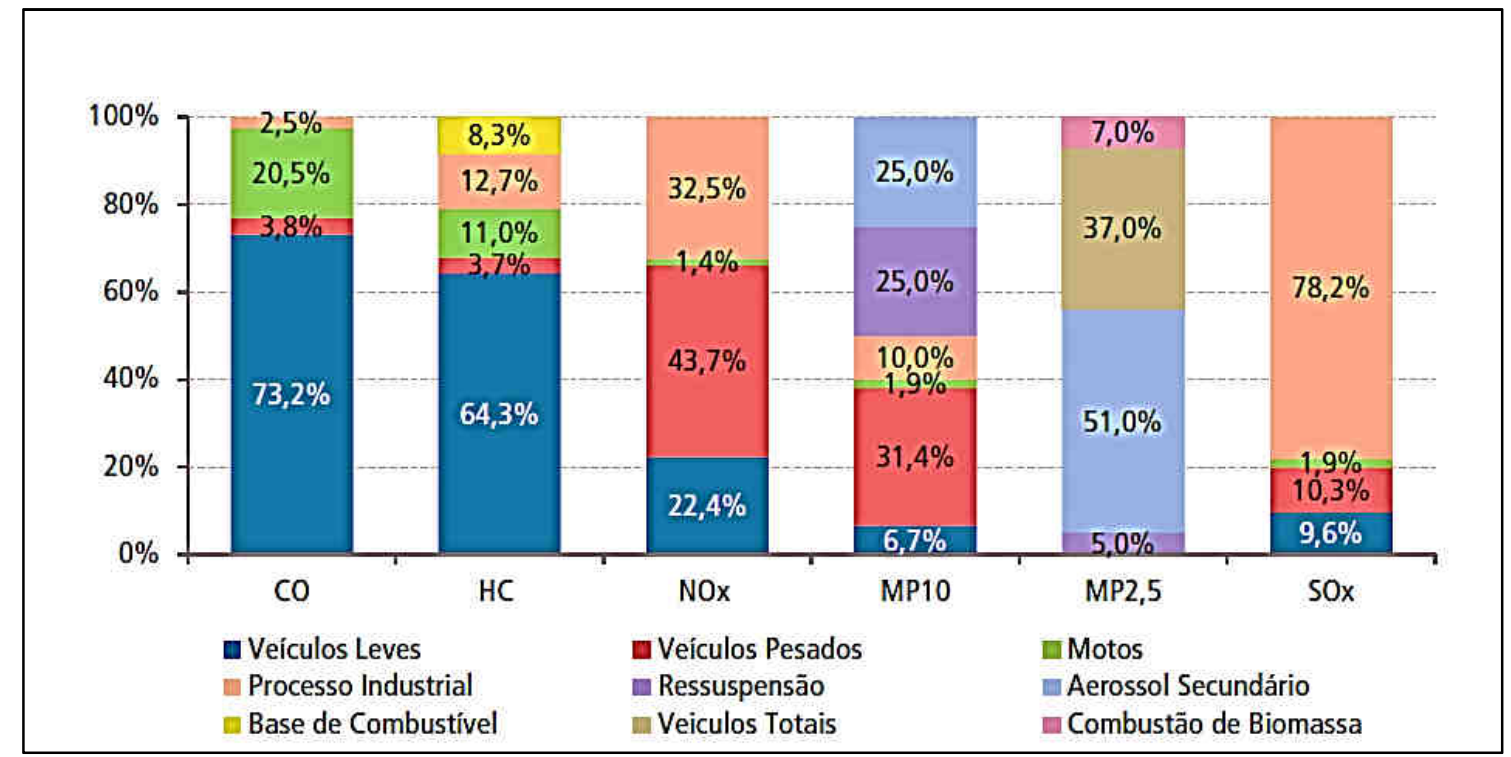

Figura 1 - Emissões relativas por tipo de fonte - RMSP [7]

A CETESB vem continuamente atuando em programas para a redução da emissão de poluentes atmosféricos; em relação às fontes fixas é desenvolvido no Estado de São Paulo o Plano para Redução de Emissão de Fontes Estacionárias (PREFE) [8]. Quanto às fontes móveis, a CETESB participa ativamente do Programa de Controle da Poluição do Ar por Veículos Automotores (PROCONVE), como agente técnico conveniado ao Instituto Brasileiro do Meio Ambiente e dos Recursos Naturais Renováveis (IBAMA), órgão do Ministério do Meio Ambiente, que gerencia este programa.

O PROCONVE é baseado em programas similares da Europa e Estados Unidos, onde o princípio fundamental é estabelecer, para os veículos novos, limites de emissão de poluentes 
que são periódica e progressivamente reduzidos, sendo comprovado o atendimento por meio de ensaios padronizados na certificação do protótipo e do acompanhamento estatístico da produção [9].

Os esforços aplicados nestes programas produziram resultados positivos, através da introdução de tecnologias para redução e controle de emissão de poluentes pelas indústrias e pelos fabricantes de veículos e motores. Assim, mesmo com o aumento da frota, houve redução significativa das emissões de $\mathrm{SO}_{2}, \mathrm{CO}$ e $\mathrm{MP}_{10}$ na RMSP e consequente melhora dos índices de qualidade do ar [6], porém ainda há altos níveis de ozônio, devido principalmente às emissões veiculares, e de $\mathrm{MP}_{2,5}$ proveniente dos veículos, ressuspensão de partículas e pela formação de aerossóis secundários [7].

O ozônio atualmente é o principal poluente atmosférico da RMSP, com grande quantidade de dias de ultrapassagem dos padrões de qualidade do ar, como mostra a Figura 2. Sua formação está fortemente influenciada pelas emissões de $\mathrm{CO}$, NOx e compostos orgânicos voláteis (COV), além de variáveis meteorológicas como índice de radiação ultravioleta, pluviosidade e intensidade dos ventos $[7 ; 10 ; 11]$. Não pode ser esquecida também a importância da emissão de gases de efeito estufa (GEE), $\mathrm{CO}_{2}$ principalmente, onde a frota veicular da RMSP é responsável pela produção de mais de 13 mil toneladas de $\mathrm{CO}_{2}$ equivalente por ano, sendo que os VLP respondem por 54\% deste total [12].

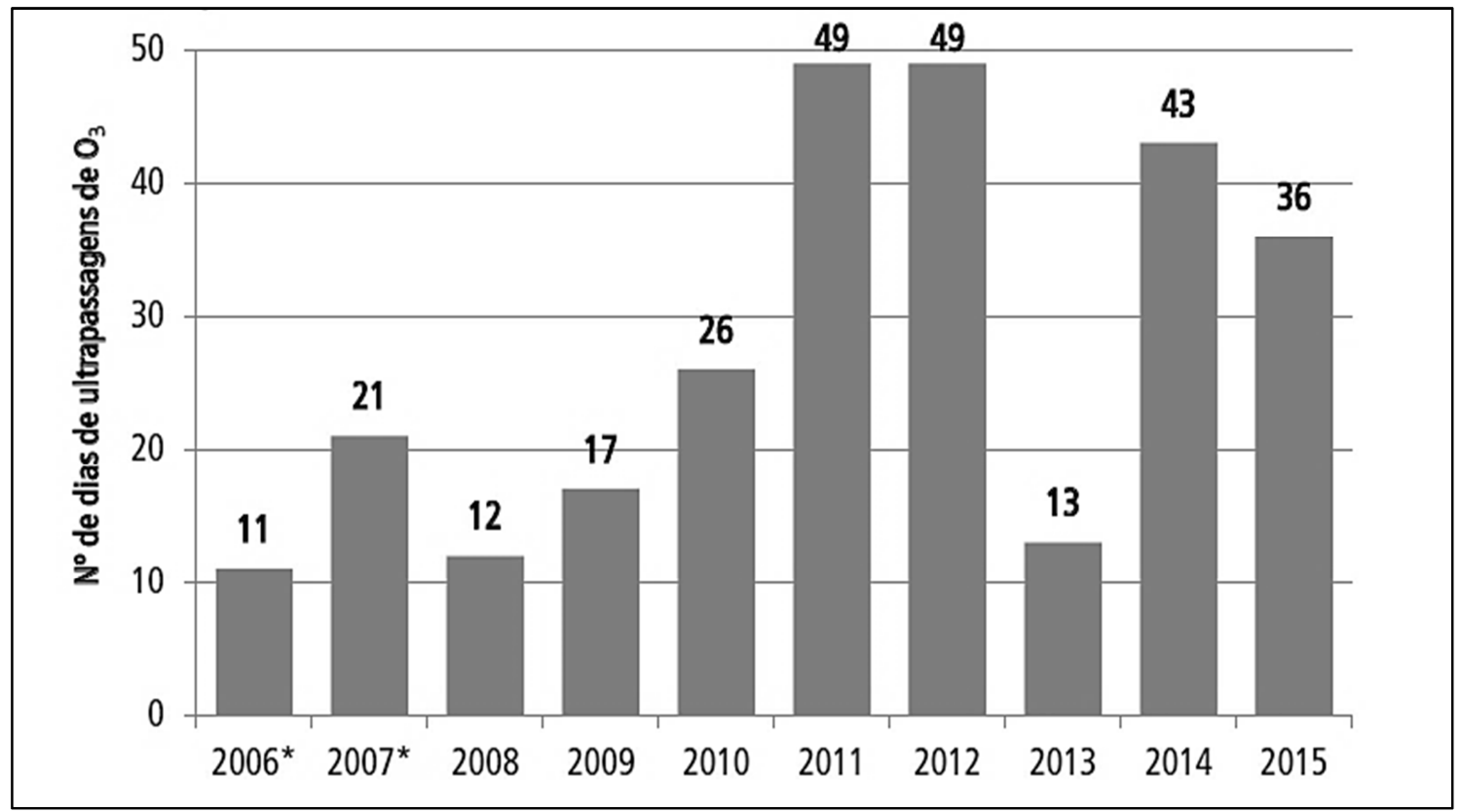

Figura 2 - Número de dias de ultrapassagem do padrão estadual de $\mathrm{O}_{3}-\mathrm{RMSP}$ [7]

O NOx tem participação importante não apenas na formação de ozônio, mas também é em si um gás poluente, que causa irritação nos olhos, nariz e mucosas, com potencial para causar doenças respiratórias como enfisema pulmonar, bronquites e câncer [13]. Outros efeitos da presença de NOx na atmosfera são a formação de particulado fino (PM2,5) e de ácido nítrico $\left(\mathrm{HNO}_{3}\right)$, que resulta em chuva ácida, além da eutrofização de corpos de água [14; 15].

\section{MÉTODO}


O processo de análise de dados consistiu em pesquisa na base de dados do setor de Homologação de Veículos (ETHV) da CETESB, coletando os resultados de emissões de $\mathrm{CO}_{2}$ e NOx em ensaios de homologação de veículos realizados no período de 2011 a 2016, portanto abrangendo as fases PROCONVE L5 e L6. Foram analisados 147 ensaios de 16 modelos diferentes de veículos leves comerciais (VLC), particularmente esportivos utilitários (SUV), vans e picapes. Cada modelo estudado deveria ter motorização similar para Diesel e gasolina e/ou flex, sendo que os critérios de similaridade utilizados foram de que os motores deveriam ter o mesmo número de cilindros, com deslocamento volumétrico e níveis de potência próximos entre si.

Esta base de dados resultou em valores típicos de emissões em ensaios de homologação para Diesel, gasolina e etanol. Também foi considerado a emissão de NOx "real", ou seja, de valores típicos obtidos em testes de rodagem em ruas (Real Driving Emissions - RDE). Diversos estudos apontam emissão de NOx de 2 a 25 vezes acima dos limites legais [16; 17; $18 ; 19 ; 20 ; 21]$; portanto, foi adotado para o NOx "real" um indexador de 4 vezes em relação ao valor obtido em testes de laboratório, baseado no estudo realizado pela CETESB e apresentado pelo IBAMA em seu endereço eletrônico [22] referente ao caso conhecido como "Dieselgate", onde foram testados veículos brasileiros.

Os dados foram então aplicados em cálculos para inventário de emissões veiculares, de acordo com a metodologia utilizada pela CETESB [12], considerando uma projeção de 6 anos de vendas de VLP a Diesel, que é a idade média da frota de VLP na RMSP, nas proporções de $50 \%$ e de $90 \%$ das vendas totais desta categoria.

\section{RESULTADOS}

Os resultados obtidos dos ensaios de homologação foram os seguintes:

Tabela 1 - Valores típicos de emissões em ensaios de homologação

\begin{tabular}{|r|c|c|c|}
\hline & Diesel & Gasolina & Etanol \\
\hline $\begin{array}{l}\mathrm{CO}_{2} \text { "bruto" } \\
\text { (sem desconto do } \\
\text { biocombustível) }\end{array}$ & $231,9 \mathrm{~g} / \mathrm{km}$ & $245,5 \mathrm{~g} / \mathrm{km}$ & $221,4 \mathrm{~g} / \mathrm{km}$ \\
\hline \multicolumn{1}{|c|}{ Diferença: } & - & $-5,5 \%$ & $+4,8 \%$ \\
\hline $\begin{array}{l}\mathrm{CO}_{2} \text { "fóssil" } \\
\text { (com desconto do } \\
\text { biocombustível - gasolina } \\
\text { com 22\% de etanol e Diesel } \\
\text { com 7\% de biodiesel) }\end{array}$ & $224,9 \mathrm{~g} / \mathrm{km}$ & $191,5 \mathrm{~g} / \mathrm{km}$ & 0 \\
\hline Diferença: & - & $+14,9 \%$ & $+100 \%$ \\
\hline NOx & $0,281 \mathrm{~g} / \mathrm{km}$ & $0,033 \mathrm{~g} / \mathrm{km}$ & $0,041 \mathrm{~g} / \mathrm{km}$ \\
\hline Diferença: & - & $+760 \%$ & $+579 \%$ \\
\hline NOx "real" & $1,124 \mathrm{~g} / \mathrm{km}$ & - & - \\
\hline Diferença: & $4 \mathrm{x}$ & - & - \\
\hline
\end{tabular}


Estes valores então, aplicados às projeções sobre os cálculos de inventário de emissões, têm como resultado:

Tabela 2 - Projeção de emissões para RMSP, com 50\% das vendas de VLP movidos a Diesel

\begin{tabular}{|c|c|c|c|}
\hline & $\mathbf{C O}_{2} \mathbf{e q}$ & NOx & NOx "real" \\
\hline $\begin{array}{c}\text { Atual } \\
\text { (mil t/ano) }\end{array}$ & 13,11 & \multicolumn{2}{|c|}{54,4} \\
\hline $\begin{array}{c}\text { Projetado } \\
\text { (mil t/ano) }\end{array}$ & 13,08 & 57,4 & 67,8 \\
\hline Diferença & $-0,3 \%$ & $+5,3 \%$ & $+19,9 \%$ \\
\hline
\end{tabular}

Tabela 3 - Projeção de emissões para RMSP, com 90\% das vendas de VLP movidos a Diesel

\begin{tabular}{|c|c|c|c|}
\hline & CO$_{2} \mathbf{e q}$ & NOx & NOx "real" \\
\hline $\begin{array}{c}\text { Atual } \\
\text { (mil t/ano) }\end{array}$ & 13,11 & \multicolumn{2}{|c|}{54,4} \\
\hline $\begin{array}{c}\text { Projetado } \\
\text { (mil t/ano) }\end{array}$ & 13,05 & 59,8 & 78,6 \\
\hline Diferença & $-0,5 \%$ & $+9,1 \%$ & $+30,9 \%$ \\
\hline
\end{tabular}

Atualmente os VLP representam 16,5\% das emissões do NOx veicular [12], porém se 90\% dos veículos leves passarem a ser movidos a Diesel, em um prazo de 6 anos se chegará a um nível de acréscimo de 24,2 mil t/ano nas emissões deste poluente, onde os VLP responderiam por $42 \%$ do total emitido, com uma redução insignificante na produção de GEE.

\section{CONCLUSÃO}

Os resultados obtidos entram claramente em confronto com a ideia que a introdução no mercado brasileiro do veículo Diesel reduziria as emissões de GEE, pelo contrário, ainda mais ao se considerar que o uso de biocombustíveis já é prática corrente dentro da nossa realidade. O aumento de NOx seria significativo, colocando os VLP a um nível de contribuição próxima à dos veículos pesados, portanto não há justificativa ambiental para se implantar esta tecnologia no Brasil, ainda mais quando se viabiliza cada dia mais alternativas como o uso de biocombustíveis e os veículos híbridos e elétricos.

\section{REFERÊNCIAS}


[1] PETROBRÁS Composição de preços ao consumidor - óleo Diesel. Disponível em: $<$ http://www.petrobras.com.br/pt/produtos-e-servicos/composicao-de-precos/diesel/> . Acesso em 06/Abril/2017.

[2] CÂMARA DOS DEPUTADOS Projeto de Lei 1013/2011. Disponível em: <http://www.camara.gov.br/proposicoesWeb/fichadetramitacao?idProposicao=498116> . Acesso em 06/Abril/2017.

[3] CETESB Posicionamento acerca do projeto de Lei $\mathbf{n}^{\circ}$ 1013, de 2011 que dispõe sobre a fabricação e venda, em território nacional, de veículos utilitários movidos a óleo diesel, e dá outras providências - Informação técnica 01/16/ETH/ET. Disponível em

$<$ http://www.cetesb.sp.gov.br/wpcontent/uploads/sites/11/2016/02/Informa\%C3\%A7\%C3\%A3o-T\%C3\%A9cnica.pdf> . Acesso em 06/Abril/2017.

[4] OMS - Organização Mundial da Saúde (WHO - World Health Organization)

International Agency for Research on Cancer - IARC: Diesel engine exhasut carcinogenic. Informação à imprensa. Lion, França: 12/Junho/2012. Disponível em: $<$ http://www.vecc-mep.org.cn/eng/news/news_detail.jsp?newsid=50712> . Acesso em 10/Abril/2017.

[5] ICCT - International Council on Clean Transportation. The European real-driving emissions regulation. Berlim, Alemanha. Disponível em: <ICCT - International Council on Clean Transportation. The european real-driving emissions regulation. Position Brief. Washington, DC, 2015. Disponível em: <http://www.theicct.org/sites/default/files/publications/ICCTbrief_EU-RDE_201512.pdf. > . Acesso em 17/Outubro/2016.

[6] CETESB - Companhia Ambiental do Estado de São Paulo. Qualidade do ar. Disponível em: 〈http://ar.cetesb.sp.gov.br/> . Acesso em 26/Novembro/2016.

[7] CETESB - Companhia Ambiental do Estado de São Paulo. Qualidade do ar no estado de São Paulo 2015. Relatório. Editoração: Roseli Arroio. São Paulo: 2016. 167 p. Disponível em: 〈http://ar.cetesb.sp.gov.br/publicacoes-relatorios/> . Acesso em 20/Maio/2016.

[8] CETESB - Companhia Ambiental do Estado de São Paulo. Plano de redução de emissão de fontes estacionárias - PREFE 2014. Coord.: Hércules Cerullo. São Paulo: 2014. 202 p.

Disponível em: <http://ar.cetesb.sp.gov.br/plano-de-reducao-de-emissao-de-fontesestacionarias-prefe/> . Acesso em 24/Novembro/2016.

[9] IBAMA - Instituto Brasileiro do Meio Ambiente e Recursos Naturais Renováveis.

Programa de controle de poluição do ar por veículos automotores -

Proconve/Promot/Ibama, 3a . Edição. Brochura. IBAMA/DIQUA: 2011. 584 p. Disponível em: 〈http://www.ibama.gov.br/areas-tematicas-qa/programa-proconve >. Acesso em 24/Junho./2015.

[10] ALVIM, D. et. al. Estudo dos compostos orgânicos voláteis precursores de ozônio na cidade de São Paulo. Artigo técnico. Engenharia Sanitária e Ambiental, Rio de Janeiro, v. 16, n. 2, 13 p., 2011. Disponível em: <http://dx.doi.org/10.1590/S1413-41522011000200013> Acesso em 21/Janeiro/2017.

[11] MARTINS, L. Sensibilidade da formação do ozônio troposférico às emissões veiculares na Região Metropolitana de São Paulo. 2006. 219 p. Tese (Doutorado em Ciências Atmosféricas). Universidade de São Paulo - Instituto de Astronomia, Geofísica e Ciências Atmosféricas, São Paulo.

[12] CETESB - Companhia Ambiental do Estado de São Paulo. Emissões veiculares no estado de São Paulo 2015. Relatório. Coord.: Marcelo Bales. São Paulo: 2016. 214 p. Disponível em: <http://veicular.cetesb.sp.gov.br/wp- 
content/uploads/sites/35/2013/12/Relatorio-Emissoes-Veiculares-2015-subst-011116.pdf> . Acesso em 23/Novembro/2016.

[13] CASTRO, A. H. S.; ARAÚJO, R. S. e SILVA, G. M. M. Qualidade do ar - parâmetros de controle e efeitos na saúde humana: uma breve revisão. Holos, ano 29, vol. 5, p. 107 a 121, Outubro/2013. ISSN: 1807-1600i

[14] EPA - United States Environmental Protection Agency. Search results for Nitrogen Oxides. Disponível em:

<https://ofmpub.epa.gov/sor_internet/registry/termreg/searchandretrieve/termsandacronyms/s earch.do?matchCriteria=Contains\&checkedTerm $=$ on\&checkedAcronym=on \&search $=$ Search \&term=Nitrogen\%20Oxides\#> . Acesso em 04/Abril/2017.

[15] ALBUQUERQUE, T. T. A. Formação e transporte das partículas finas inorgânicas em uma atmosfera urbana: o exemplo de São Paulo. Tese (Doutorado em Metereologia). 2010, 189 p. Universidade de São Paulo - Instituto de Astronomia, Geofísica e Ciências Atmosféricas, São Paulo.

[16] FRANCO, V. et al. Real-world exhaust emissions from modern diesel cars - A metaanalysis of PEMS emissions data from EU (EURO 6) and US (Tier 2 bin 5/ulev II) Diesel passenger cars. Part 1: aggregated results. Berlim, Alemanha: ICCT - International Council on Clean Transportation Europe, 2014, 59 p. Disponível em: $<$ http://www.theicct.org/sites/default/files/publications/ICCT_PEMS-study_dieselcars_20141010.pdf>. Acesso em 04/Janeiro/2017.

[17] KADIJK, G. et al. TNO 2016 R10083 NOx emissions of Euro 5 and Euro 6 diesel passenger cars - test results in the lab and on the road. Relatório. Delft, Holanda: TNO Netherlands Organisation for Applied Scientific Research, 2016, 33 p. Disponível em: <http://publications.tno.nl/publication/34620046/H95fkX/TNO-2016-R10083.pdf > . Acesso em 23/Setembro/2016.

[18] GERMAN, J. The emissions test defeat device problem in Europe is not about VW.

Berlim, Alemanha: ICCT - International Council on Clean Transportation Europe, 2016, 8 p. Disponível em: <http://www.theicct.org/blogs/staff/emissions-test-defeat-device-problemeurope-not-about-vw $>$, acesso em 25/Novembro/2016.

[19] KODJAK, D. et al. An international perspective on vehicle emissions compliance, testing and enforcement. In: US EPA COMPLIANCE SUMMIT, 2016, Ann Arbour, Estados

Unidos. Anais... Estados Unidos: EPA, 2016, 22 p.

[20] MARNER, B. Emission of nitrogen oxides from modern Diesel vehicles. Relatório. Bristol, UK: Air Quality Consultants, 2016. 48 p. Disponível em:

$<$ http://www.aqconsultants.co.uk/getattachment/Resources/Download-Reports/Emissions-ofNitrogen-Oxides-from-Modern-Diesel-Vehicles-210116.pdf.aspx > . Acesso em

23/Setembro/2016.

[21] THOMPSON, G. et al. In-Use Emissions Testing of Light-Duty Diesel Vehicles in the United States - final report. Morgantown, Estados Unidos: CAFEE - Center for Alternative Fuels, Engines \& Emissions - West Virginia University, 2014, 133 p. Disponível em: $<$ http://www.theicct.org/sites/default/files/publications/WVU_LDDV_inuse_ICCT_Report_Final_may2014.pdf>. Acesso em 11/Janeiro/2017.

[22] IBAMA - Instituto Brasileiro do Meio Ambiente e dos Recursos Naturais Auto de infração n 9082389-E - Procedimento 02001.007032/2015-46 - Decisão de $1^{a}$ instância 191/2017 - Parecer DIQUA 000207/2017 - Relatório de avaliação de emissões de poluentes de veículos Amarok Diesel, CETESB, 01.2017. Auto de infração. Brasília, DF: 21/março/2017. Disponível em: <http://www.ibama.gov.br/phocadownload/noticias/noticias2017/oficio_volkswagen_e_anexo s_.pdf>. Acesso em 06/Abril/2017. 